\title{
The Non-Uniform Spatial Development of a Micrometastasis
}

\author{
MALCOLM I. G. BLOOR and MICHAEL J. WILSON* \\ Department of Applied Mathematics, The University of Leeds, Leeds LS2 9JT, UK
}

(Received 11 May 1998; Revised 26 October 1998; In final form 29 January 1999)

\begin{abstract}
This paper presents a mathematical model for the growth of a cancer micrometastasis in the form of a vascular cuff. The model postulates the possibility of a local imbalance between the rate of cell proliferation and the rate of cell death through apoptosis which is taken as dependent on the concentration of an angiogenesis-inhibitor such as angiostatin. This imbalance produces non-zero cell velocities within the micrometastasis. The local cell velocity is related to an interstitial pressure gradient through a Darcy's Law type of equation, and the spatio-temporal development of the micrometastasis in an environment with a non-uniform nutrient concentration is followed by treating its outer boundary as an advancing front.
\end{abstract}

Keywords: Cancer, metastasis, tumour, apoptosis, angiostatin

\section{INTRODUCTION}

Metastasis, the release of cancer cells from a primary tumour and their spread to other parts of the body, is the major cause of mortality in cancer patients [20]. The transport of malignant cells to distant parts of the body is predominantly carried out by the vascular and lymphatic systems, although it can also occur through direct physical contact, e.g. as a result of surgical activity.

Metastasis can be regarded as comprising three stages: Invasion, embolization, and extravasion [23]. Invasion of the tissue surrounding a primary tumour is a fundamental property of cancer cells, and along with the overall processes of metastasis, distinguishes the cells of malignant from benign tumours. The process of invasion is the result of a complex sequence of events which involves the detachment of tumour cells from the primary tumour, their attachment to the basement membrane matrix, the degradation of that matrix, the locomotion and infiltration of tumour cells into the region where the matrix has broken down, the degradation of the extracellular matrix, and finally the degradation of the vascular basement membrane matrix and the invasion of the lymphatic or blood vascular channel [23]. Some mathematical modelling of this stage of metastasis has been carried out by Perumpanani et al [37] who, taking into account the interaction of malignant cells with normal cells,

\footnotetext{
${ }^{*}$ Corresponding author.
} 
other tumour cells, and the extracellular matrix, use a travelling wave approach to model invasion.

Embolization is the entry into and transport of tumour cells through the vascular system. Even though considerable numbers of cells may be released into the vascular system, apparently only a very small fraction $(<1$ in 10,000$)$ are able to survive the process and establish secondary tumours elsewhere in the body. The reasons why the proportion of cancer cells surviving this process should be so low are not well understood, but may be connected with the need of normal cells of a particular tissue type to adhere to one another and to the appropriate extracellular matrix, or else die through apoptosis. This property, which prevents cells of normal tissue from floating away and establishing themselves elsewhere in the body, appears to be absent from at least some cancer cells, so that although most embolized tumour cells may still die through apoptosis, a fraction can survive to become established elsewhere in the body [36].

Circulating tumour cells usually become trapped in the first vascular bed that they encounter downstream of the primary tumour. The next step in the establishment of a secondary tumour, is extravasion which again involves a complex sequence of events. Briefly, however, the cancer cell attaches itself at some point in a vascular bed, effects the retraction of the endothelial cells at that point, migrates through the breach in the vessel wall, degrades the surrounding matrix and establishes a metastatic tumour. Since the first vascular bed encountered by blood leaving most organs belongs to the lungs, it is here that the most common site for metastasis can be found [23]. Metastatic tumour cells may form microscopic perivascular 'cuffs' around a capillary at the location where originally a clonogenic cell left the circulation $[20,17]$. In the lungs of a mouse, such cuffs are no larger than about $150 \mu \mathrm{m}$ [17], or about ten cells [20], in radius.

A micrometastasis may remain in a dormant avascular state with a high cell proliferation rate balancing a high cell death rate by apoptosis, a form of programmed cell death which is usually characterized by single-cell death in the midst of living cells $[41,40,6]$. The onset of angiogenesis can be triggered by the removal of the primary tumouralthough Folkman [17] notes that for certain other mouse tumours micrometastases may not become angiogenic, i.e. the angiogenesis genes are not activated, even 3.5 months after removal of the primary tumour.

In a previous paper [7] the authors presented a mathematical model of a micrometastasis which described the growth of a cylindrically symmetric micrometastasis around a central capillary out of which nutrient was diffusing. The model assumed that growth initially took place because of a local imbalance between cell proliferation and apoptotic cell death, with proliferation exceeding death close to the capillary. However, due to a decrease in the nutrient concentration with radial distance away from the capillary and a consequent decrease in the proliferation rate, at larger distances from the capillary the relative importances of proliferation and death were reversed, and consequently the micrometastasis tended towards a 'dormant' state with a finite outer radius.

This original model assumed the geometry for the micrometastasis to be that of a circular cylinder, so that only growth in the radial direction was produced. This paper presents a refinement to the model so that, although the micrometastasis is still growing around a cylindrical capillary, only axial symmetry is assumed, i.e. growth can now take place parallel to the capillary wall as well as in the radial direction. Furthermore, the new model also takes into account the effects produced by an axial variation of nutrient concentration - and consequently proliferation rate - as well as a radial variation.

In the earlier work [7] a time-varying apoptic death rate was considered in order to model the effect of the angiogenesis inhibitor angiostatin upon the micrometastasis. This work was prompted by a series of papers in which the effect of angiostatin upon human and murine primary tumours implanted in a mouse model was investigated, as was its effect upon the secondary metastases which the implanted primary produced $[30,20,31]$. It was reported that treatment with angiostatin was capable 
of regressing the primary tumour and limiting the metastases to a microscopic dormant state. Evidence indicated that these effects were achieved through an elevation in the apoptotic death rate, and that angiogenesis, if unhindered by a substance such as angiostatin, significantly lowered apoptosis in metastases although their high cell proliferation rate remained unaltered [20].

On the basis of this work O'Reilly et al [31] suggest a new paradigm for anticancer treatment based upon the administration of anti-angiogenesis agents such as angiostatin - although the possibility of this type of approach to anti-cancer therapy had been suggested in the early 1970 s by Folkman [16]. Research into the effects of angiostatin upon primary and metastatic cancer has continued, e.g. $[21,42,38]$, and indeed a new angiogenesisinhibitor called endostatin has been identified and its effects investigated [32]. In addition, Boehm et al [8] show that treatment with endostatin caused a primary tumour implanted within a mouse model to regress. If the treatment with endostatin discontinued the tumours regrew, however upon resumption of the treatment with endostatin the tumours could be successfully re-regressed - a cycle which could be repeated. As with earlier work with angiostatin, the work on endostatin indicated that the inhibition of angiogenesis seemed to result in a high-rate of apoptosis in tumours cell in treated mice compared to the rate in tumour cells in untreated mice, whereas there was no difference in the proliferation rate of the tumour cells in the two sets of mice.

Below we present results which indicate the effect of increasing the apoptotic death-rate at some stage during the time-development of a micrometastasis, in order to model the effects of an angiogenesisinhibitor.

\section{THE MATHEMATICAL MODEL}

Most mathematical modelling of the metastatic process has concentrated upon the initial stages in which cancer cells are lost from the primary tumour, invade the surrounding tissue, and then enter the circulatory or lymphatic systems. For instance, Chaplain and Sleeman $[13,14]$ consider the growth of a solid primary tumour using non-linear elasticity theory in particular membrane and thick-shell theory. Using this approach they are able to give conditions in terms of the strain energy function for the processes of invasion and metastasis to occur. Adam [3] has described how catastrophe theory may provide a qualitative description of the growth of metastases.

The process by which a primary tumour becomes vascularized is important from the point of view of metastasis since the presence of nearby capillaries greatly enhances the capacity of tumour cells to spread to other parts of the body. Orme and Chaplain [33] have considered the growth of new capillaries towards a primary tumour and the advance of invading tumour cells towards regions of high blood vessel density. In a later paper [34], also concerned with the modelling of tumour angiogenesis, they model the effects of an antiangiogenesis drug on the tumour vasculature. As mentioned above, the process of invasion of the surrounding tissue by tumour cells has been considered Perumpanani et al [35] who describe a continuum model for the behaviour of the invasive cells that takes into account their interactions with normal cells, noninvasive tumour cells, and ECM proteins and proteases.

The aspect of the problem we consider here is that of a perivascular cuff growing around a capillary vessel of radius $r_{m}$, where we assume axial symmetry in the model so that, if $z$ is the coordinate direction along the capillary and $r$ is the radial coordinate measured from the centre of the capillary, the radial position $R$ of the outer boundary of the tumour is a function of $z$ and $t$.

In common with other mathematical models for the growth of solid tumours, e.g. $[11,15,18,19,24,1$, $14,39]$, we adopt a continuum approach in that the properties of the tumour cells are modelled in an aggregative sense, not at the level of individual cells. The discrete nature of the problem does not alter the underlying physical processes, and thus a continuum model captures perfectly adequately the overall growth of the cuff without any loss of physical insight. In fact, although experiment, as 
mentioned above, indicates that micrometastases are about 10 cells in radius, they can be many hundreds of cells long.

We assume that the cancer cells obtain nutrient, in this case oxygen, by diffusion from the capillary which they surround, and as a result they divide and proliferate. Like other mathematical models for tumour growth a mechanism for the loss of cell volume is assumed, which in this case is apoptosis, a form of programmed cell death [40]. Apoptosis as a mechanism for the loss of cell volume has been considered in the context of spherical tumours by McElwain and Morris [25] and Byrne and Chaplain $[9,10]$, who found that it was possible to obtain a dormant state even in the absence of a nonnecrotic core.

At any point in the microscopic tumour, there will be a local volume proliferation rate $k(r, z)$ (cell volume created/unit volume/unit time), and a local apoptotic volume death rate $\alpha(r, z)$ (cell volume destroyed/unit volume/unit time). Any local imbalance in proliferation and apoptosis will produce a non-zero cell velocity $\underline{u}$ at that point, where

$$
\nabla \cdot \underline{u}=(k-\alpha)
$$

where it has been assumed that after apoptotic death a cell ceases to occupy any volume. Thus, an inevitable consequence of the original model, indeed of almost any model for tumour growth, is a movement of cells through the tumour.

In this model we assume that cell proliferation and cell death produce internal pressure differences responsible for the cell motion, and that in their motion the cells behave like an incompressible fluid for which the viscous forces experienced by the cells are much smaller than the inertial forces. In these circumstances we would expect that the flux of cellular material would be proportional to the pressure gradient, thus following Greenspan [19], McElwain et al [26], and McElwain and Pettet [27], we assume that the cell velocity $\underline{u}$ is related to the local internal pressure $p$ by a Darcy's Law type of equation [5]:

$$
\underline{u}=-\mu \nabla p
$$

where the pressure $p$ and velocity $u$ are interpreted as average values.

As in the previous model [7], the evolution of the micrometastasis is tracked by following the motion of its boundary $R(z, t)$. This is done by noting that there will be no relative motion between an 'element' of tumour lying on the boundary and the boundary itself. Thus, following an element in the free surface, the rate of change of $R$ is equal to the radial component of that element's velocity for all time, and the usual free-surface condition follows in the form:

$$
\frac{\partial R}{\partial t}+u_{z} \frac{\partial R}{\partial z}-u_{r}=0
$$

where $u_{r}$ and $u_{z}$ are the radial and axial components of $\underline{u}$ at points on the surface of the micrometastasis (see for example Lighthill [22]). The cell velocity $\underline{u}$ is obtained from equation (2) while the pressure distribution within the tumour is obtained by solving

$$
\mu \nabla^{2} p=-(k-\alpha)
$$

Thus, in a sense, the growth of the micrometastasis is driven by the right-hand side of equation (4) which gives the local imbalance between cell proliferation and cell death, and this imbalance is related to the local nutrient concentration, in this case oxygen.

The diffusion of nutrient out from the central capillary we assume takes place on a much shorter time-scale than the typical time-scales for cell proliferation and death (a natural assumption to make, see for example Adam [2]), so that we have a quasisteady model in which the oxygen concentration $c$ obeys the diffusion equation

$$
\frac{D}{r} \frac{\partial}{\partial r}\left(r \frac{\partial c}{\partial r}\right)+D \frac{\partial^{2} c}{\partial z^{2}}=s
$$

where $s$ is the rate of consumption of oxygen per unit volume per unit time, and $D$ is the diffusion coefficient of oxygen, both assumed to be constant whilst the cells of the micrometastasis are viable.

We will assume that the length-scale for the variation of $c$ with $r$ is much shorter than the lengthscale for the variation of $c$ with $z$, and so, retaining 
the dominant term, the equation effectively obeyed by the nutrient concentration is

$$
\frac{D}{r} \frac{\partial}{\partial r}\left(r \frac{\partial c}{\partial r}\right)=s
$$

where, the above assumption clearly allows a 'slow' variation of $c$ with $z$.

For the micrometastasis being considered, there is no evidence that the cancer cells are becoming less viable due to nutrient starvation. Furthermore, we argue below that the radius of the micrometastasis $r_{m}$ is much small than its diffusion limited size $r_{e}$, so that the consumption of nutrient by the micrometastasis will be negligible and hence the variation in nutrient concentration will largely be a result of diffusion into the surrounding normal tissues. Thus we use a nutrient concentration at the central capillary which reflects the fact that nutrient is constantly being lost to the surrounding tissue and hence which decreases as one moves along the capillary.

Given that the nutrient concentration at the capillary wall $c_{0}(z)$ will vary with distance $z$ as a result of the overall transfer of nutrient to the body, the spatial variation of $c(r, z)$ can be described by an expression of the form

$$
\begin{aligned}
c(r, z) & =c_{0}(z)-\frac{1}{D}\left(m(z)+\frac{s r_{m}^{2}}{2}\right) \ln \left(r / r_{m}\right) \\
& +\frac{s}{4 D}\left(r^{2}-r_{m}^{2}\right)
\end{aligned}
$$

where the oxygen concentration at the capillary wall is $c_{0}(z)$, the radius of the capillary is $r_{m}$, and the nutrient flux $\left(-D r_{m}(\partial c / \partial r)\right)$ is $m(z)$.

As previously [7], we will assume that the nutrient concentration $c$ experienced by the micrometastasis never gets so low that necrosis occurs, which means that its radius must remain much smaller than $(2 m(z) / s)^{1 / 2}$, which is the radius at which $r(\partial c / \partial r)=0$ (assuming $r_{m}$ to be small), i.e. the radius, $r_{e}$, the micrometastasis would need to be if it were to consume all the nutrient flux from the central capillary. Typically we would expect $r_{e}$ to be orders of magnitude larger than $r_{m}$; for example, spherical tumours to become diffusion-limited at a radius of around 200 cells.
Putting $r_{e}^{2}(z) \approx((2 m(z)) / s)$, equation $(7)$ can be written

$$
\begin{aligned}
c(r)= & c_{0}(z)-\frac{s}{2 D}\left(r_{e}^{2}(z)+r_{m}^{2}\right) \ln \left(r / r_{m}\right) \\
& +\frac{s}{4 D}\left(r^{2}-r_{m}^{2}\right)
\end{aligned}
$$

and, if we assume that throughout its growth the radius $r$ of the micrometastasis remains much smaller than $r_{e}$, i.e. $r_{m}<r \ll r_{e}(z)$, the oxygen concentration the micrometastasis experiences can be approximated by

$$
c(r)=c_{0}(z)-\frac{s r_{e}^{2}(z)}{2 D} \ln \left(r / r_{m}\right)
$$

since the third term on the right-hand side of equation (8) remains smaller than the second for $r \ll r_{e}$. In other words the amount of nutrient consumed by the cancer cells is a small fraction of the total amount available, i.e. there is no significant variation in the oxygen concentration due to consumption by the micrometastasis.

In common with other models, see for example McElwain and Ponzo [24], McElwain and Morris [25], Byrne and Chaplain [9,10], it is assumed here that the cell proliferation rate is a function of nutrient concentration which for simplicity we take to be of the form $k \propto$ (constant + constant $c$ ), which, given equation (9), implies that in this case

$$
k(r, z)=k_{0}(z)-k_{2}(z) \ln \left(r / r_{m}\right)
$$

with $k_{0}(z), k_{2}(z)>0$.

In the absence of experimental evidence, we make the simplest possible assumption concerning the rate of apoptosis $\alpha$ which we initially take to be a constant throughout the micrometastasis at a given moment. Obviously, a variation in $\alpha$ could be put into the model if experimental evidence demanded it. Note that for the micrometastasis to start to grow, we require $k_{0}(z)>\alpha$.

\section{TUMOUR EVOLUTION}

To use the model to calculate the growth of the micrometastasis we proceed as follows. Starting 
with an initial shape for the micrometastasis and a given nutrient concentration, we can obtain the pressure distribution within the tumour by solving equation (4). In the results presented in this paper, this was done using a finite-volume approach on a polar mesh that continuously adapts as the micrometastasis grows. The source-term on the right-hand side of (4), which is a function of position, is obtained from the form of the nutrient concentration. Thus the form of $k(r, z)$ is given by equation (10), although, of course, one must still choose the constants which relate $k$ to $c$. The particular choice for $k(r, z)$ is discussed below in Section (4).

The boundary of the region over which equation (4) is solved is the unknown outer surface of the micrometastasis and the interface between the micrometastasis and the capillary wall, i.e. at $r=r_{m}$. The pressure distribution within the medium around the tumour is assumed to be uniform, whilst the cell velocity normal to the capillary wall is zero. Hence equation (4) is solved subject to the following boundary conditions:

$$
\begin{gathered}
p=0 \text { on the outer boundary } \\
\quad \text { of the tumour, } R(r, z, t) \\
\frac{\partial p}{\partial r}=0 \text { at the capillary wall, } r=r_{m}
\end{gathered}
$$

Having obtained the pressure distribution within the micrometastasis, the velocity field can be obtained from equation (2) by finite-differencing the pressure distribution.

Finally, the velocity distribution on the surface of the micrometastasis can be used with the equation (3) to update the position of the tumour boundary, which was again solved using finitedifferences. Note that the finite-differencing of the time derivatives is used to evolve the surface of the tumour over the time-interval $\Delta t$ used in the temporal finite-difference, i.e. $R(r, z, t) \rightarrow R(r, z, t+$ $\Delta t$ ). Note also that movement of the outer boundary of the metastasis usually requires a constant remeshing of the enclosed volume in order to solve equation (4) with the necessary accuracy.
This procedure is repeated over successive timesteps to follow the growth of the tumour for as long as is required.

\section{RESULTS}

We start the metastasis evolution with an almost spherical tumour of radius $4 r_{m}$ centred on $z=0$, the deviation from perfect sphericity being due to the presence of the capillary.

We present results for the following functional form for the proliferation rate

$$
k(r, z)=\left(k_{0}-k_{1} \tanh \left(2 \frac{z}{z_{0}}\right)\right)\left(1-k_{2} \ln \left(r / r_{m}\right)\right)
$$

which is equivalent to assuming a nutrient concentration given by

$$
c(r, z)=c_{0}-c_{1} \tanh \left(2 \frac{z}{z_{0}}\right)\left(1-g 2 \ln \left(r / r_{m}\right)\right)
$$

where $c_{0}, c_{1}, z 0$ and $g 2$ are constants. This form for the concentration variation models the effect of removing oxygen from the blood and hence in decreasing the nutrient concentration as one moves along the capillary in the direction of the flow. As noted above, the consumption of nutrient by the micrometastasis is insignificant, the oxygen concentration is essentially determined by its diffusion into the mass of normal tissue surrounding the capillary. In using equation (14) we have taken a functional variation of $c(r, z)$ that satisfies the diffusion equation (6) and, as seems reasonable, models a decrease in nutrient concentration as one moves along the capillary. $\mathrm{A} \tanh (z)$ variation has been used as a convenient means of producing a smooth variation from an upstream value to a lower downstream value of the nutrient concentration, with the length-scale determined by $z_{0}$. Obviously, another functional variation with this general property could have been used so long as it satisfied the requirement for a slow variation in the $z$-direction.

We are assuming that the flow of blood along the capillary is in the direction of increasing $z$. Thus 
for $z \ll 0$, upstream of the capillary, the nutrient concentration is essentially constant; for $z \simeq 0$ the nutrient concentration decreases essentially linearly with $z$; whilst for $z \gg 0$, well downstream of the metastasis, the nutrient concentration is constant once again, but with a lower value. Refering to the constants in equation (13) we can see that they have the following significance: $k_{0}$ represents the mean of the upstream and downstream proliferation rates; $2 k_{1}$ represents the drop in proliferation rate of the cells adjacent to the capillary wall due to reducing nutrient concentration in the blood; $z_{0}$ determines the axial length-scale over which the proliferation rate drops; whilst $k_{2}$ gives the length-scale of the radial variation in proliferation rate.

In the earlier version of this model [7] it was shown that an appropriate scaling for the time variable was obtained from the proliferation rate, so that here we introduce a non-dimensional time variable, defined thus

$$
\tau=k_{0} t
$$

to scaie the time evolution of the tumour. Hence in all the results presented below showing the growth of the tumour, the time is given in units of $1 / k_{0}$, which is a measure of the mean celldoubling time (in the absence of apoptosis). Note that for tumour cells the net doubling-time (with apoptosis present) is of the order of a week [20]. The equation giving the 'net' proliferation rate can thus be non-dimensionlised as follows

$$
\begin{aligned}
k(r, z)-\alpha= & \left(1-\gamma \tanh \left(2 \frac{z}{z_{0}}\right)\right) \\
& \times\left(1-k_{2} \ln \left(r / r_{m}\right)\right)-\eta
\end{aligned}
$$

where we have introduced the following dimensionless parameters

$$
\begin{aligned}
& \eta=\frac{\alpha}{k_{0}} \\
& \gamma=\frac{k 1}{k_{0}}
\end{aligned}
$$

where $\eta$ characterizes the relative importance of apoptosis to proliferation, while $\gamma$ characterizes the fractional drop in the proliferation rate as one moves along the capillary. The parameter $k_{2}$ in equations (13) and (16) is already dimensionless and determines the radius $r_{m} e^{1 / k_{2}}$ at which proliferation falls to zero due to the radial decrease in nutrient concentration, i.e. it is closely related to $r_{e}$.

The assumption that the length-scale for the $z$ variation in the nutrient concentration is larger than the length-scale for $r$ variations, means that we require $z_{0} \gg r_{m}$. In all results presented here, the parameter $z_{0}$, has been taken to be $20 r_{m}$. Furthermore, the natural length-scale to choose is $r_{m}$ so that in the results presented below, all distances are given in units of $r_{m}$.

We take the parameter $\mu$, which relates the cell velocity to the internal pressure gradients via equation (2) to be unity, since this constant merely scales the pressure $p$.

Notice that we can determine whether or not the micrometastasis grows at all by considering $(k-\alpha)$ in the region adjacent to the capillary wall. In particular at $r=r_{m},(k-\alpha)$ varies between $1+$ $\gamma-\eta$ and $1-\gamma-\eta$ in non-dimensional units, so that for the micrometastasis to grow at all we require $1+\gamma>\eta$, and for it to penetrate into the downstream region we require $1-\gamma>\eta$.

Below are presented results which give an indication of the main 'regimes' of micrometastatic growth. The results are given in three forms: as a series of images which show the micrometastasis at various stages in its development; as a graph which shows the maximum radial dimension ( $r \max$ ), and the maximum and minimum axial positions ( $\mathrm{zmax}$ and $\mathrm{zmin}$ ) of the micrometastasis, as functions of time; and, for those with $\mathrm{CD}$ rom facilities, as animated QuickTime 'movies' of the tumour growth. The images and movies have been generated on a Silicon Graphics workstation.

\subsection{Asymmetric Axial Growth Rate}

Figures (1) and (2) show the results for the following set of parameters: $\left(\eta=0.5, \gamma=0.5, k_{2}=0.23\right)$. Figure (1) shows the tumour at various selected times, while Figure (2) shows a graph of its radial and axial dimensions as functions of time. For this 
$t=16$

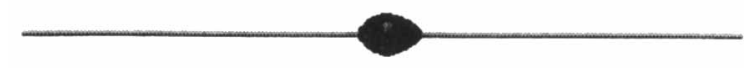

$t=34$

$t=44$

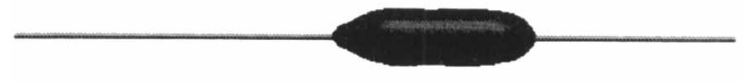

$t=55$

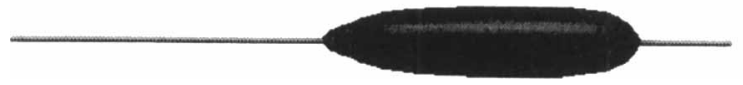

FIGURE 1 Asymmetric growth $\left(\eta=0.5, \gamma=0.5, k_{2}=0.23\right)$.

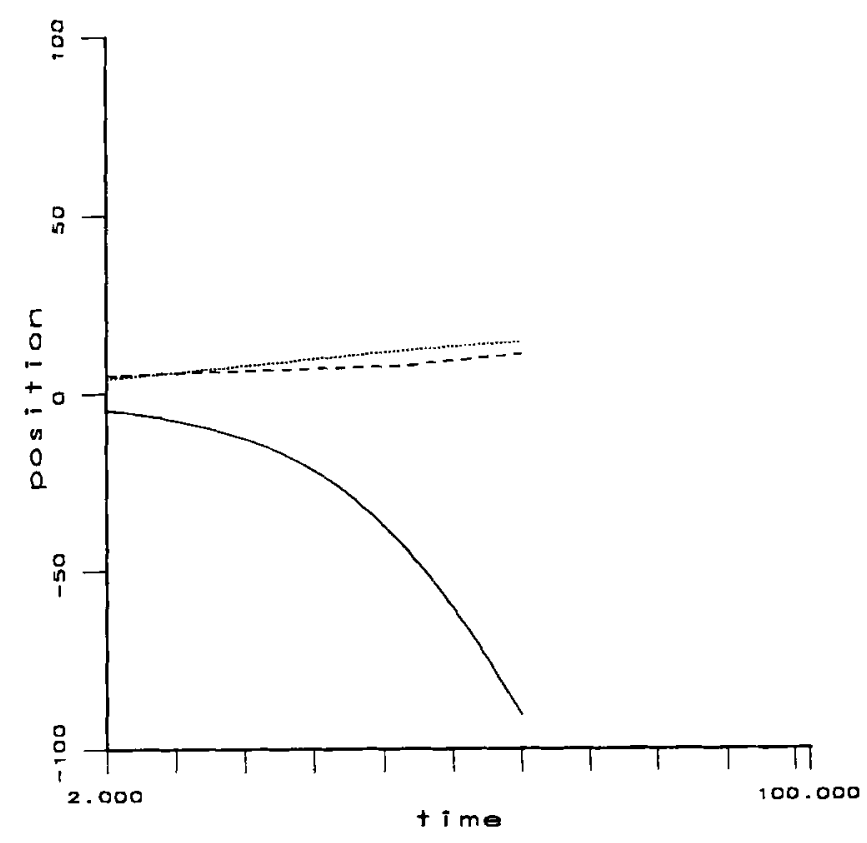

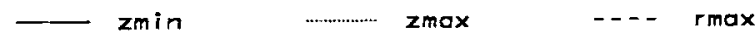

FIGURE 2 Tumour dimensions for asymmetric growth $\left(\eta=0.5, \gamma=0.5, k_{2}=0.23\right.$ ). 
choice of parameters, in particular the non-zero value of the parameter $\gamma$, the axial variation in the nutrient concentration, and hence the proliferation rate $k$, results in a strong asymmetry in the axial growth of the micrometastasis, with the tumour growing preferentially in the direction of increasing nutrient concentration. In particular, for this choice of parameters $1-y=\eta$, so that the micrometastasis cannot grow far in the downstream direction. Note also that the growth in the opposite direction and also the radial growth of the micrometastasis is limited by a balance between proliferation $k$ and apoptosis $\alpha$, i.e. $k \sim \alpha$.

A QuickTime movie demonstrating tumour growth can be viewed from the on-line paper and archive CD-ROM of this journal.

\subsection{Symmetric Axial Growth Rate}

In the next set of results, shown in Figures (3) and (4), the parameter $\gamma$ has been set to zero so that there is no longer any axial variation in the nutrient concentration and hence no variation in the proliferation rate. As might be expected the tumour now grows at equal rates in opposite directions along the capillary. The radial growth of the tumour is limited by the logarithmic fall with $r$ of the proliferation rate, so that the radial extent of the micrometastasis is limited by a balance between proliferation and apoptosis in its outer regions.

A QuickTime movie demonstrating tumour growth can be viewed from the on-line paper and archive CD-ROM of this journal.

$$
t=26
$$

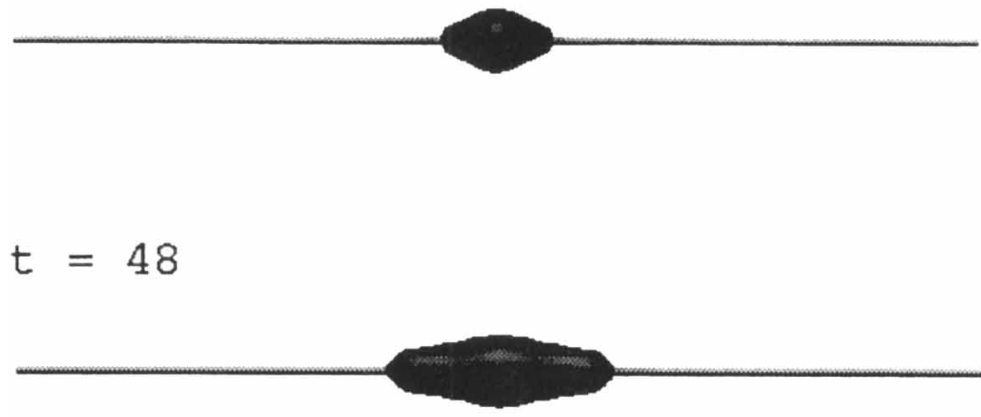

$t=82$

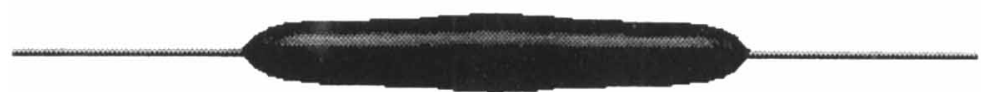

$t=102$

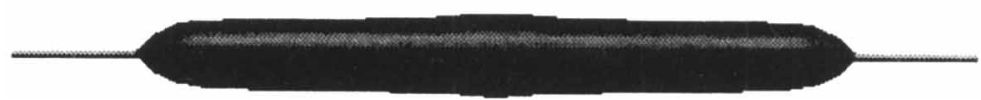

FIGURE 3 Symmetric axial growth $\left(\eta=0.5, \gamma=0.0, k_{2}=0.23\right)$. 


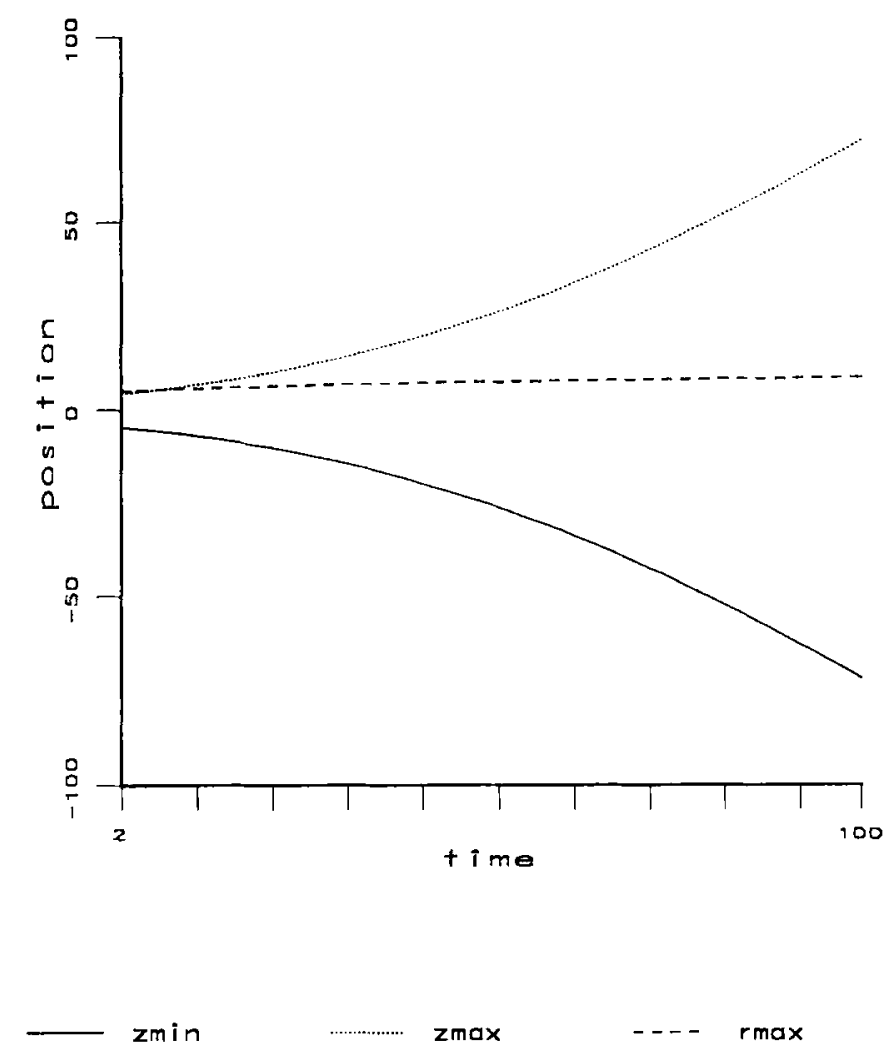

FIGURE 4 Tumour dimensions for symmetric axial growth $\left(\eta=0.5, \gamma=0.0, k_{2}=0.23\right)$.

\subsection{Radial Growth}

In Figures (5) and (6) both $\gamma$ and $k_{2}$ have been set to zero, so that there is no longer any axial or radial variation in the nutrient concentration. Strictly speaking this violates the assumption which enables us to ignore the second term of equation (5), however this case has been included for the sake of comparison.

As expected the growth of the tumour is moreor-less spherical, although not exactly so. The slight deviation from spherical symmetry is due to a growth rate along the capillary that is slightly lower than elsewhere. This is explicable on the basis that cancer cells adjacent to the capillary wall will experience lower axial pressure gradients since there are cells proliferating on only one side of them.

A QuickTime movie demonstrating tumour growth can be viewed from the on-line paper and archive CD-ROM of this journal.

\subsection{Asymmetric Axial Growth with Decreased Apoptosis}

Finally, in Figures (7) and (8) the original set of parameters have been used but with $\eta=0.1$, so that the apoptotic death rate has been reduced. In this case the axial growth of the micrometastasis is again strongly asymmetric and the radial growth of the micrometastasis eventually becomes limited by apoptosis. Now, however, since the apoptotic death rate is lower, the micrometastasis can expand further in the radial direction before the proliferation rate drops to such a level where it becomes comparable with apoptosis and radial growth stops.

A QuickTime movie demonstrating micrometastasis growth can be viewed from the on-line paper and archive CD-ROM of this journal.

For the sake of comparison the relative growth rates of the results above are shown in Figures (9) and (10) which are a graph of the logarithm of the 


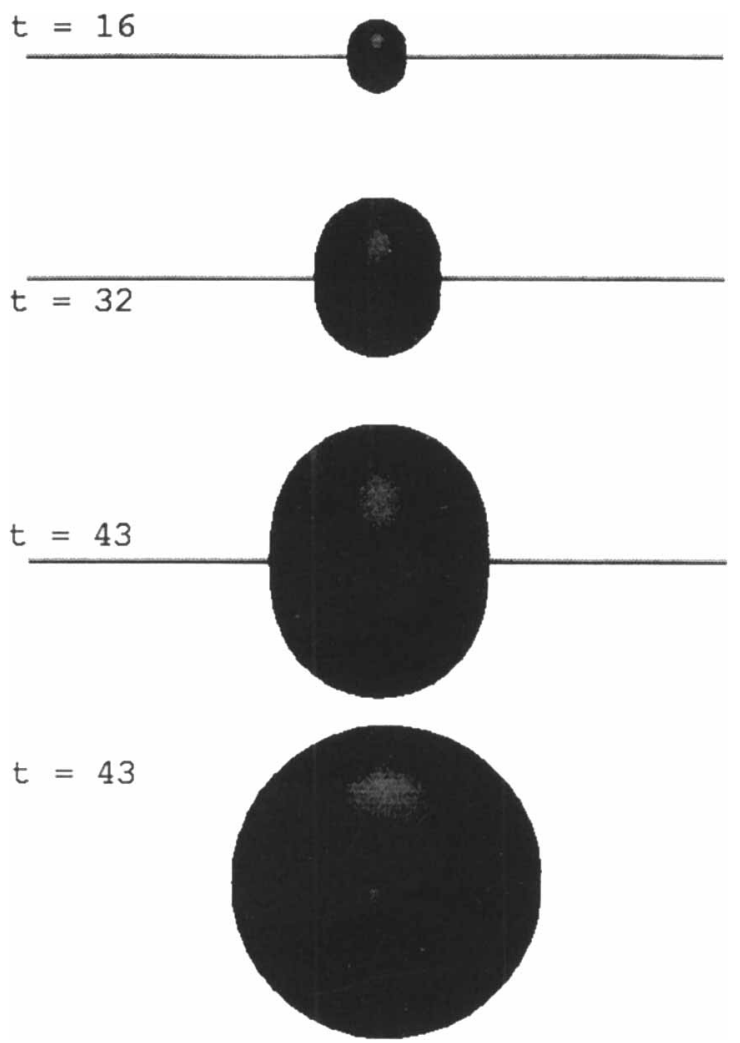

FIGURE 5 Radial growth $\left(\eta=0.5, \gamma=0.0, k_{2}=0.0\right)$.

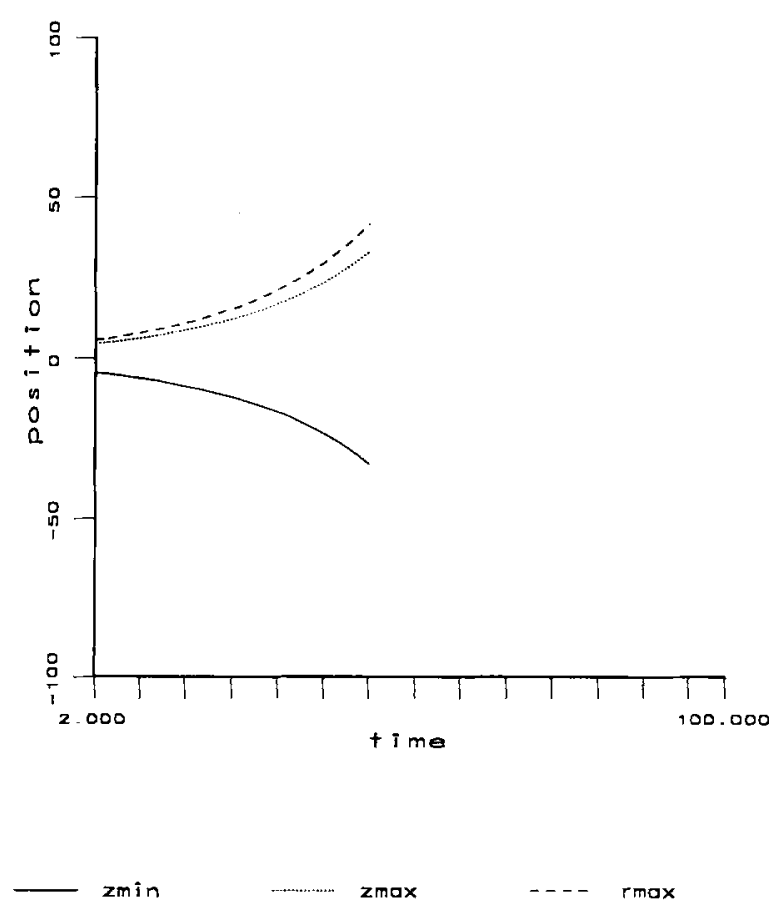

FIGURE 6 Tumour dimensions for radial growth $\left(\eta=0.5, \gamma=0.0, k_{2}=0.0\right.$ ). 
$t=18$
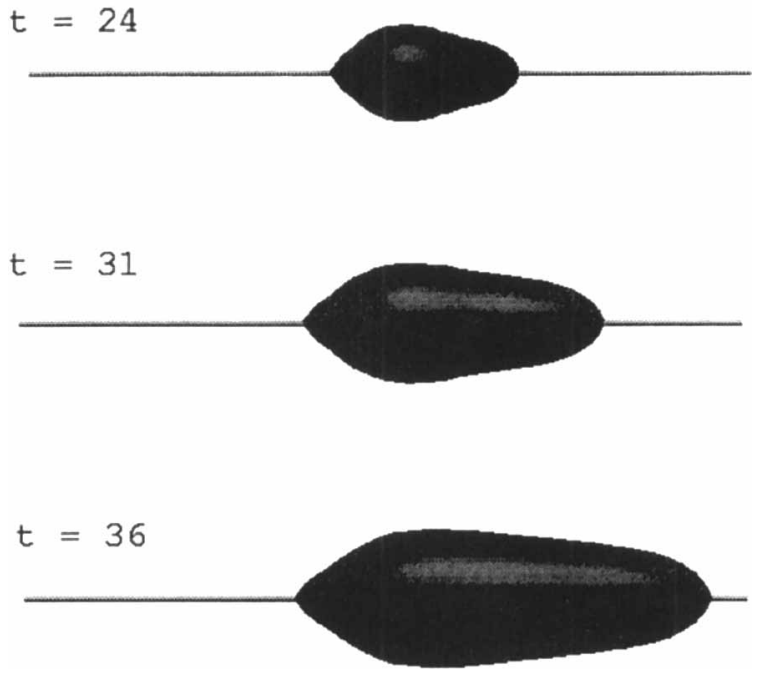

FIGURE 7 Axial growth with decreased apoptosis $\left(\eta=0.1, \gamma=0.5, k_{2}=0.23\right)$.

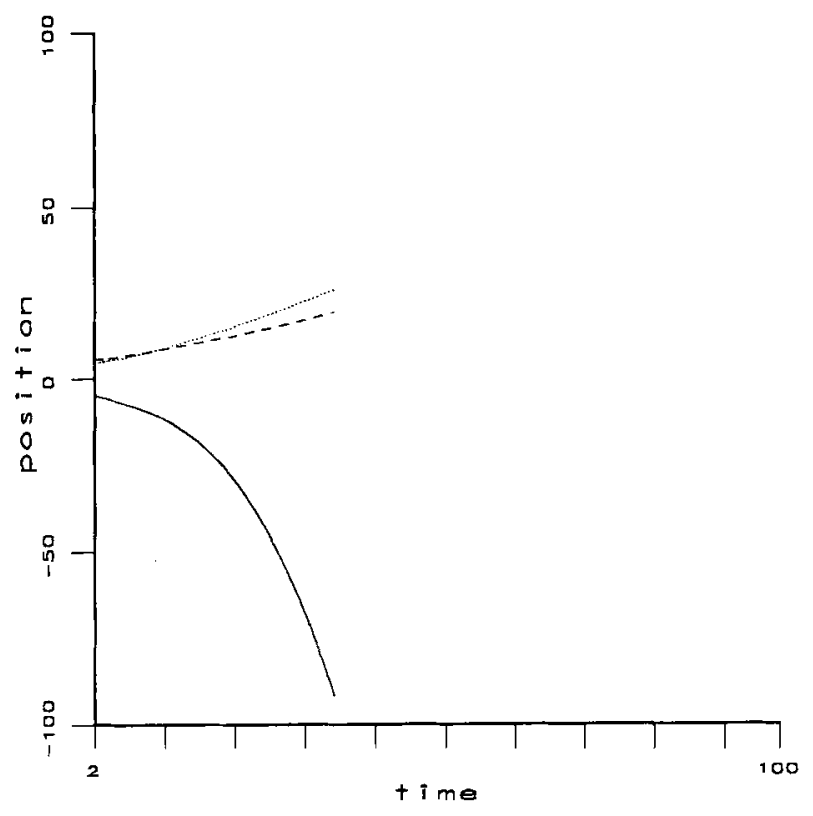

zmin $\quad$ zmin.......... zmax $\quad$ max

FIGURE 8 Tumour dimensions for asymmetric axial growth $\left(\eta=0.1, \gamma=0.5, k_{2}=0.23\right)$. 

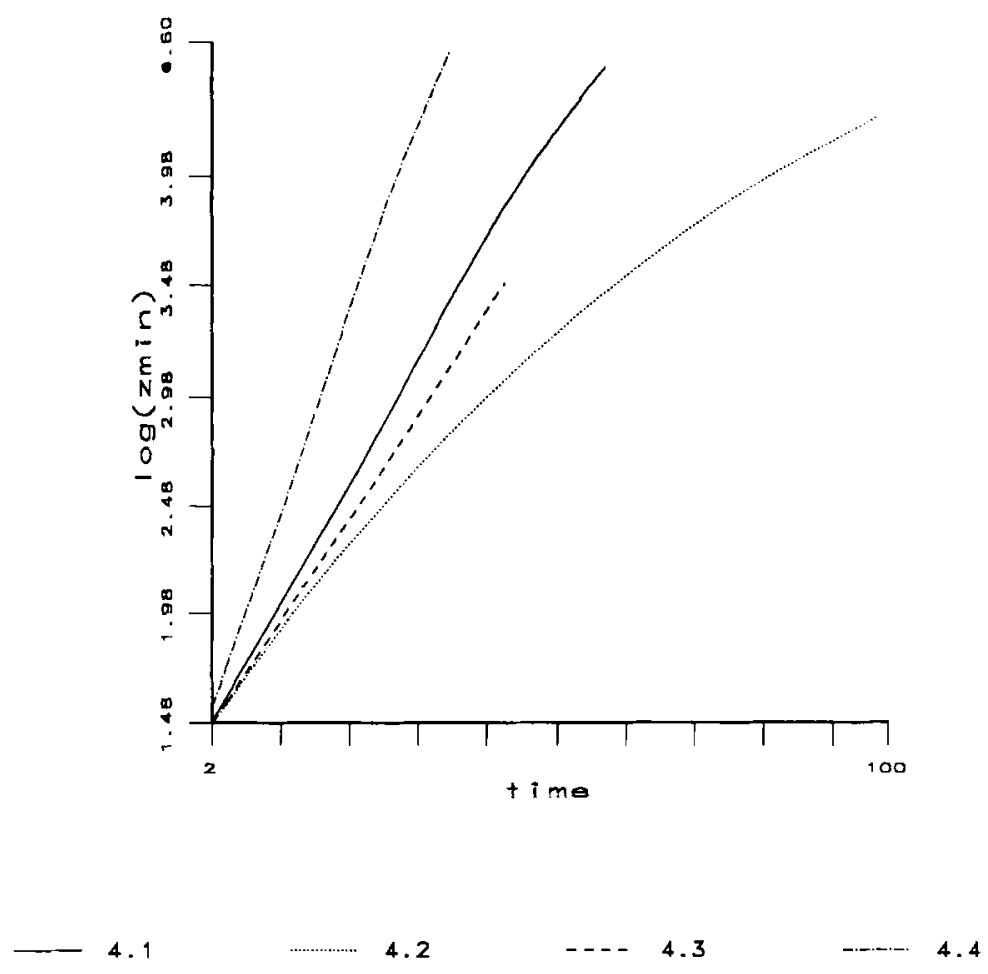

FIGURE 9 Logarithmic variation of tumour dimensions.

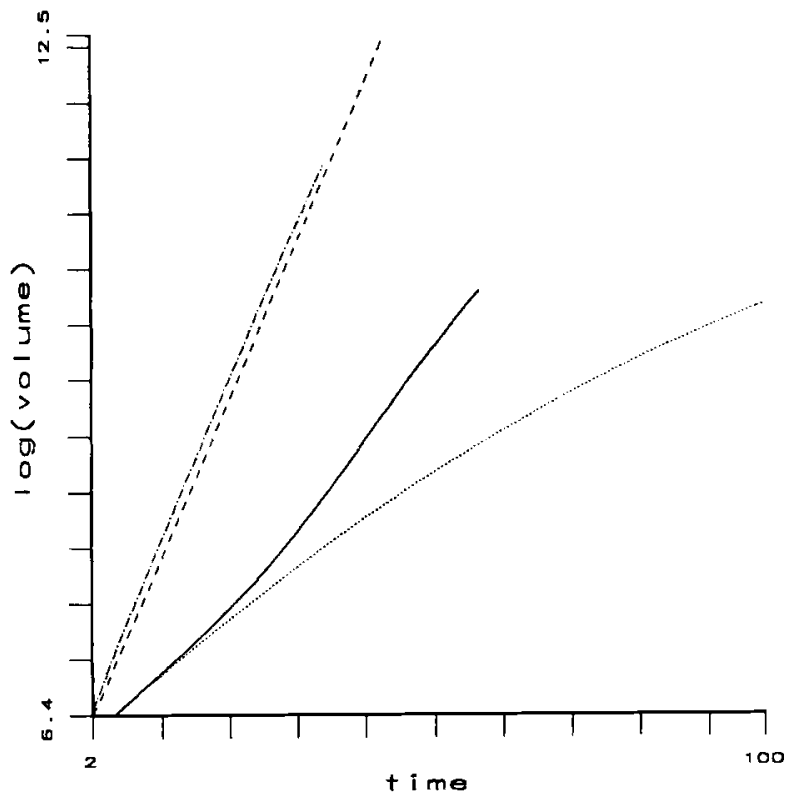

4. 1
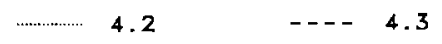

-... 4.4

FIGURE 10 Logarithmic of tumour volume. 
fastest growing linear dimension (usually $\mathrm{zmin}$ ) against time, and a graph of the logarithm of the tumour's volume, respectively. In all four cases on can see that the growth is initially exponential, but then starts to fall below this as time progresses. One can also see that for the same rate of apoptosis, the greatest rate of volume increase occurs when there is no radial or axial variations in the proliferation rate, so that the growth is approximately spherical. Interestingly, the volume of the micrometastasis in 4.1 grows faster than that in 4.2 , even though there is no axial variation in proliferation rate for 4.2 . Finally, the large growth rate in 4.4 can be attributed to the lower value of the apoptotic death-rate than in the preceding runs.

\subsection{Tumour Regression due to Increased Apoptosis}

In this section we consider the effects of increasing the apoptotic death-rate in order to model the possible effects of raising Angiostatin or Endostatin concentration at some stage in the development of the micrometastasis. As mentioned in the introduction, it has been suggested that these substances can produce tumour regression by elevating the apoptotic death-rate [20]. At present, there is no experimental evidence to give the quantitative relationship between the angiostatin concentration and the apoptotic death-rate. Therefore in the absence of such data, we consider a uniform increase in the death rate $\alpha$ as the first term in the functional relationship.

For the sake of example we consider two cases, where initially in both cases the micrometastases develop as described in Section (4.4), i.e. $(\eta=0.1$, $\gamma=0.5, k_{2}=0.23$ ), up to time $t=31$. At this point, in the first case the value of $\eta$ is raised to 1.0 , while in the second the value of $\eta$ is raised to 1.3. The results of the first case are shown in Figure (11) while the results of the second are shown in Figure (12); both figures show the micrometastases at selected times, starting at the point where the apoptotic death-rate is raised.

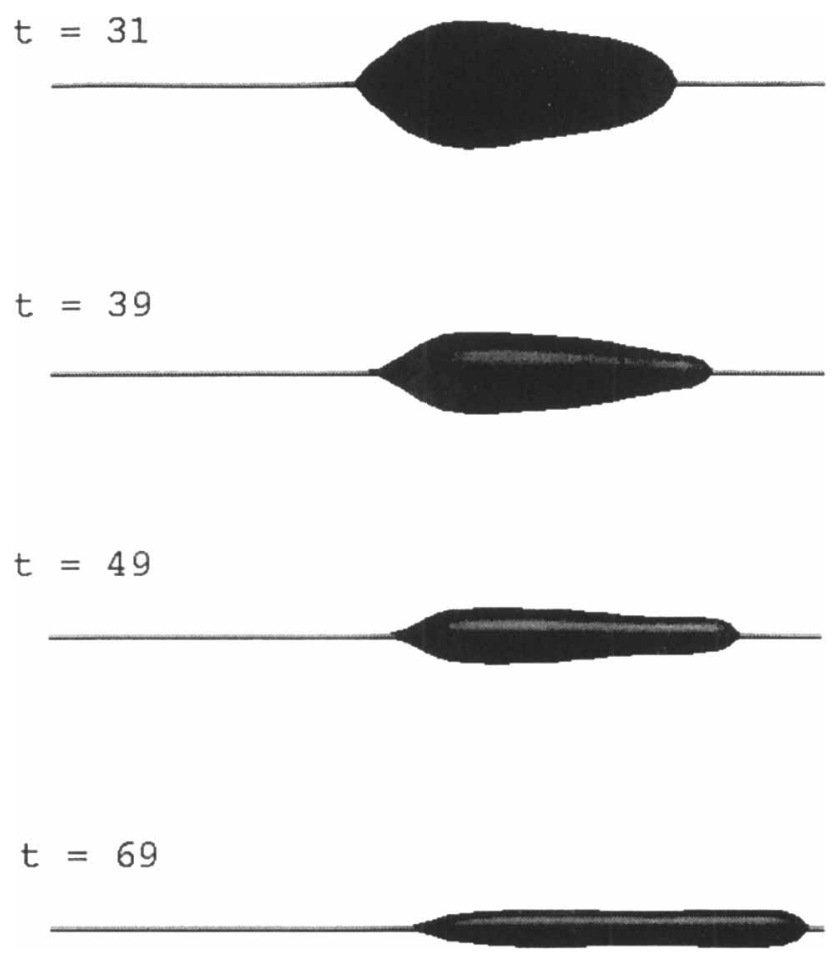

FIGURE 11 Axial growth with increased apoptosis $\left(\eta_{\text {old }}=0.1, \eta_{\text {new }}=1.0, \gamma=0.5, k_{2}=0.23\right)$. 


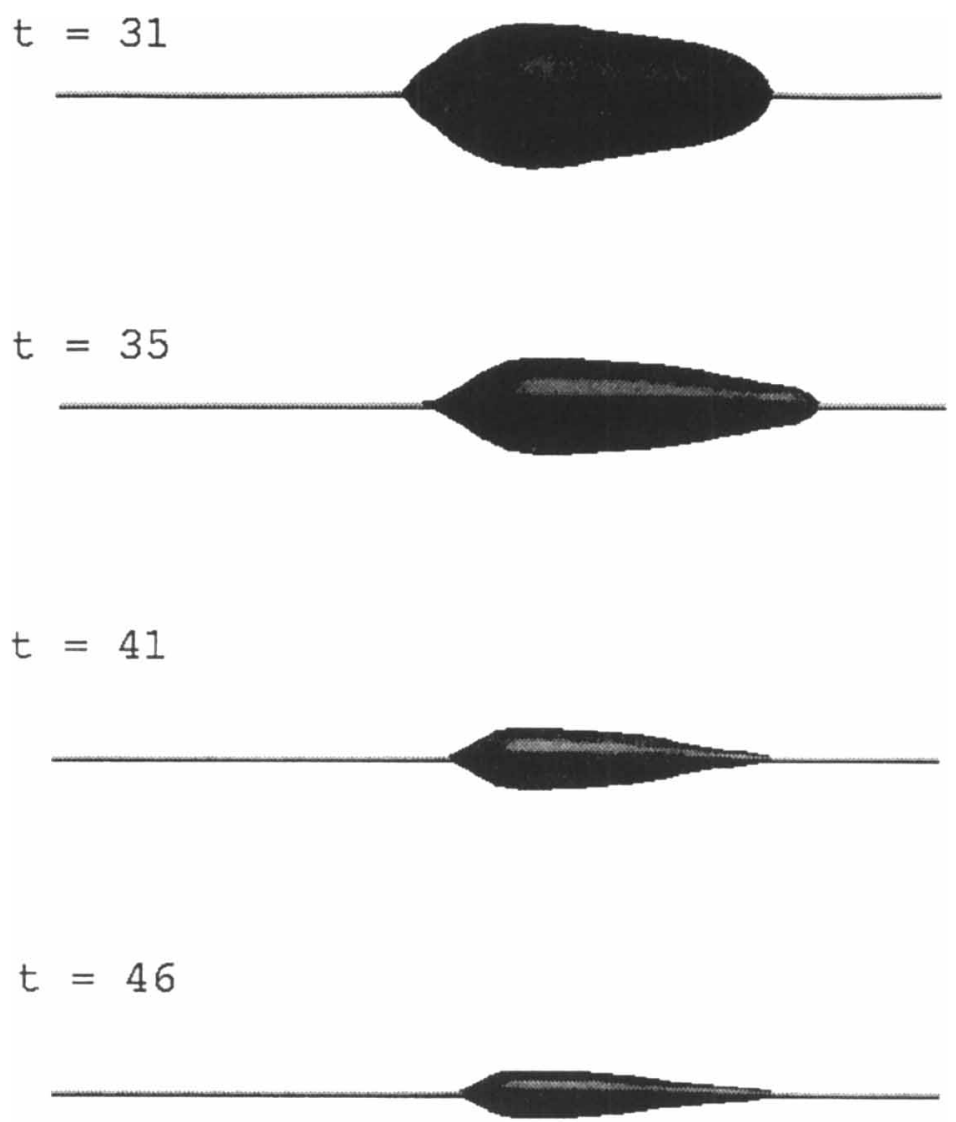

FIGURE 12 Axial growth with increased apoptosis $\left(\eta_{\text {old }}=0.1, \gamma=0.5, k_{2}=0.23\right.$ ).

For the micrometastasis shown in Figure (11), $1+\gamma>\eta_{\text {new }}$ whereas $1-\gamma<\eta_{\text {new }}$. The first inequality indicates that the micrometastasis can grow in the upstream direction, whilst the second indicates that it cannot grow in the downstream. These conclusions are borne out by reference to Figure (11), where it can be seen that in response to the increase in the death-rate the radius of the micrometastasis shrinks and yet it continues to grow in the upstream direction. In other words, the level of the angiogenesis-inhibitor is too low to completely halt the growth.

A QuickTime movie demonstrating micrometastasis growth can be viewed from the on-line paper and the archive CD-ROM of this journal.

For the micrometastasis shown in Figure (12), $1+\gamma<\eta_{\text {new }}$, which indicates that after the increase in the death-rate the tumour cannot grow at all, in fact it must regress. This is clearly shown in Figure (12) where the micrometastasis rapidly shrinks in size in both the radial and axial directions. Since $1-\eta<0$ everywhere within the tumour, i.e. the death-rate exceeds the proliferation rate, it will eventually disappear, although it is laborious to follow this process to the very end using the numerical scheme employed in this paper.

A QuickTime movie demonstrating micrometastasis shrinkage can be viewed from the on-line paper and the archive CD-ROM of this journal.

\section{CONCLUSIONS}

Following on from earlier work [7] we have presented a model for the spatio-temporal development of a micrometastasis growing around a central 
capillary. The original model assumed a cylindrical tumour, but in this paper this assumption has been relaxed to allow for more general axisymmetric growth. This has been achieved by assuming that the velocity $u$ of tumour cells within the tumour is related to the interstitial pressure $p$ by a Darcy's Law type of equation (2), and by solving a nonlinear free-surface equation in order to track the boundary of the tumour as an advancing front. By assuming a proliferation rate that depends on the nutrient concentration and by considering different possible variations of the nutrient concentration, various regimes of growth have been demonstrated.

It has been demonstrated that the result of treatment with an anti-angiogenesis agent such as angiostatin or endostatin $[30,31,32]$ can easily be taken into account in the present model, where tumour regression can be modelled just as easily as tumour progression, and as more information becomes available this aspect of the model can be refined. This was illustrated by the results presented in Section (4.5), where it was shown that only if the increase in the death-rate is large enough did the micrometastasis regress, whereas in other situations, although the radial extent of the micrometastasis will decrease and it may regress on its downstream side, it can continue to grow along the central capillary towards regions of high nutrient concentration.

The model can be extended in a number of ways and adapted as more information becomes available through experiment. Perhaps the most obvious is to extend it to three-dimensional growth to take into account growth along a network of capillaries for example. This would mean calculating the solution to equation (2) on a three-dimensional mesh and also extending equation (3) to describe the motion of an advancing surface in three-dimensions, rather than an advancing curve in two as is presently the case. Other ways to extend the model would be to use more detailed modelling of the nutrient variation in the capillary bed, and/or to use a more realistic functional relation between the nutrient concentration and the proliferation rate. Furthermore, it was here assumed that the apoptotic death-rate is constant throughout the micrometastasis. The model could be modified to include the effects of a spatial dependence in the concentration of the angiogenesis-inhibitor combined with a realistic dependence of the death-rate upon the concentration of the inhibitor. In addition, the time-decay in the concentration of the angiogenesis-inhibitor in the central capillary could be included, an effect which has already been modelled to some extent by Adam and Bellomo [4] who considered the post-surgical effects of removal of the primary tumour.

Although the model is presented here in the context of modelling micrometastasis, it is also of relevance to other aspects of cancer modelling. For example, the model can be applied to the non-uniform growth of solid tumours in general, in particular the process of tumour vascularization and the resulting growth. Also the development of tumour cords found in a number of human and animal tumours, which are cylindrical cuffs that separate central blood vessels from areas of necrosis $[28,29]$ can be addressed in a similar way.

\section{References}

[1] Adam, J. A. (1991). Diffusion models of prevascular and vascular tumour growth, in Mathematical Population Dynamics, Lecture notes in pure and applied mathematics, Marcel Dekker, New York, pp. 625-652.

[2] Adam, J. A. and Noren, R. D. (1993). Equilibrium model of a vascularized spherical carcinoma with central necro sis - some properties of the solution, J. Math. Bio., pp. $735-745$.

[3] Adam, J. A. (1996). Mathematical models of prevascular spheroid development and catastrophe-theoretic description of rapid metastatic growth tumour regemission, Invasion and Metastasis, 16(4-5), pp. 247-267.

[4] Adam, J. A. (1997). Post-surgical passive response of local environment to primary tumour removal, Mathematical and Computer Modelling, 25(6), pp. 7-17.

[5] Batchelor, G. K. (1973). An Introduction to Fluid Dynamics, Cambridge University Press, Cambridge.

[6] Bellamy, C. O. C., Malcolmson, R. D. G., Harrison, D. J. and Wyllie, A. H. (1995). Cell death in health and disease: The biology and regulation of apoptosis, Seminars in Cancer Biology, 6, pp. 3-16.

[7] Bloor, M. I. G. and Wilson, M. J. (1997). A Mathematical Model of a Micrometastasis, J. Theo. Med., 1(2), pp. 153-168.

[8] Boehm, T., Folkman, J., Browder, T. and O'Reilly, M. S. (1997). Antiangiogenic therapy of experimental cancer does not induce acquired drug resistance, Nature, 390, pp. $404-407$. 
[9] Byrne, H. M. and Chaplain, M. A. J. (1995). The growth of nonnecrotic tumours in the presence and absence of inhibitors, Math. Biosci., 130, pp. 151-181.

[10] Byrne, H. M. and chaplain, M. A. J. (1996). The growth of necrotic tumours in the presence and absence of inhibitors, 135, pp. 187-216.

[11] Burton, A. C. (1996). "Rate of growth of solid tumours on a problem of diffusion", Growth, 30, pp. 157-176.

[12] Chaplain, M. A. and Sleeman, B. D. (1990). A mathematical model for the production and secretion of tumour angiogenesis factor in tumours, IMA J. Math. Appl. Med. Bio., 7, pp. 93-108.

[13] Chaplain, M. A. and Sleeman, B. D. (1992). A mathematical model for the growth and classification of a solid tumour - a new approach via nonlinear elasticity theory using strain-energy functions, Math. Biosciences, 111, pp. $169-215$

[14] Chaplain, M. A. and Sleeman, B. D. (1993). Modelling the growth of solid tumours and incorporating a method for their classification using nonlinear elasticity theory, J. Math. Bio., 31, pp. 431-473.

[15] Deakin, A. S. (1975). Model for the growth on an in vitro tumour, Growth, 39, pp. 155-165.

[16] Folkman, J. (1972). Annals of Surgery, 285, pp. 409-416.

[17] Folkman, J. (1995). Angiogenesis in cancer, vascular, rheumatoid and other disease, Nature Medicine, 1(2), pp. 27-31.

[18] Greenspan, H. P. (1972). Models for the growth of a solid tumour by diffusion, Stud. Appl. Math., 52, pp. 317-340.

[19] Greenspan, H. P. (1976). On the growth and stability of cell cultures and solid tumours, J. Theo. Bio., 56, pp. 229-242.

[20] Holmgren, L., O'Reilly, M. S. and Folkman, J. (1995). Dormancy of micrometastases: Balanced proliferation and apoptosis in the presence of angiogenesis suppression, Nature Medicine, 1(2), pp. 149-153.

[21] Lannutti, B. J., Gately, S. T., Quevedo, M. E., Soff, G. A. and Paller, A. S. (1997). Human Angiostatin inhibits Murine Hemangioendothelioman Tumour Growth, Cancer Research, 57, pp. 5277-5280.

[22] Lighthill, M. J. (1978). Waves in Fluids, Cambridge University Press, Cambridge.

[23] Mellors, R. C. (1995). Neoplasia, Cornell University Medical College Pathology Notes, Cornell University Medical College Internet pages, http://edcenter.med.cornell.edu/ CUMC_PathNotes/Neoplasia/Neoplasia_TOC.html.

[24] McElwain, D. L. S. and Ponzo, P. J. (1977). A model for the growth of a solid tumour with non-uniform oxygen consumption, Math. Biosciences, 35, pp. 267-279.

[25] McElwain, D. L. S. and Morris, L. E. (1978). Apoptosis as a volume loss mechanism in mathematical models of solid tumour growth, Math. Biosciences, 39, pp. 147-157.

[26] McElwain, D. L. S., Callcott, R. and Morris, L. E. (1979). A model of vascular compression in solid tumours, $J$. Theo. Bio., 78, pp. 405-415.

[27] McElwain, D. L. S. and Pettet, G. J. (1993). Cell migration in multicell spheroids: Swimming against the tide, Bull. Math. Bio., 55(3), pp. 655-674.
[28] Moore, J. V., Hopkins, H. A. and Looney, W. B. (1984). Tumour-cord parameters in two rat hepatomas that differ in their radiobiological oxygenation status. Radiat. Environ. Biophy., 23, pp. 213-222.

[29] Moore, J. V., Haselton, P. S. and Buckley, C. H. (1985). Tumour cords in 52 human bronchial and cervical squamous cell carcinomas: Inferences for their cellular kinetics and radiobiology. Br. J. Cancer, 51, pp. 407-413.

[30] O'Reilly, M. S., Holmgren, L., Shing, Y., Chen, C., Rosenthal, R. A., Moses, M., Lane, W. S., Chao, Y., Sage, E. H. and Folkman, J. (1994). Angiostatin: A novel angiogenesis inhibitor that mediates the suppression of metastases by a Lewis Lung Carcinoma, Cell, 79, pp. 315-328.

[31] O'Reilly, M. S., Holmgren, L., Chen, C. and Folkman, J. (1996). Angiostatin induces and sustains dormancy of human primary tumours in mice, Nature Medicine, 2(6), pp. 689-692.

[32] O'Reilly, M. S., Boehm, T., Shing, Y., Fukai, N., Vasios, G., Lane, W. S., Flynn, E., Birkhead, J. R., Olsen, B. R. and Folkman, J. (1997). Endostatin: An endogeneous inhibitor of angiogenesis and tumor growth, Cell, 88, pp. 277-285.

[33] Orme96, M. E. and Chaplain, M. A. J. (1996). A mathematical model of vascular tumour growth and invasion, Mathematical and Computer Modelling, 23(10), pp. 43-60.

[34] Orme96, M. E. and Chaplain, M. A. J. (1997). Twodimensional models of tumour angiogenesis and antiangiogenesis strategies, IMA Journal of Mathematics applied in Medicine and Biology, 14(3), pp. 189-205.

[35] Perumpanani, A. J., Sherratt, J. A. and Norbury, J. (1997). Biological inferences from a mathematical model for malignant invasion, Invasion and Metastasis, 16(4-5), pp. 209-221.

[36] Ruoslahti, E. (1996). How Cancer Spreads, Scientific American, September, pp. 42-47.

[37] Perumpanani, A. J., Sherratt, J. A., Norbury, J. and Byrne, H. M. (1996). Biological Inferences from a Mathematical Model for Malignant Invasion, Invasion and Metastasis, 16, pp. 209-221.

[38] Sim, B. K. L., O'Reilly, M. S., Liang, H., Fortier, A. H., Weixuan, H., Madsen, J. W., Lapcevich, R. and Nacy, C. A. (1997). Human Angiostatin inhibits Murine Hemangioendothelioma Tumor Growth, Cancer Research, pp. $1329-1334$

[39] Sleeman, B. D. (1996). Solid Tumour Growth: A Case Study in Mathematical Biology, in Nonlinear Mathematics and its Applications, ed. Philip, J. A., Cambridge University Press, pp. 237-256.

[40] Steller, H. (1995). Mechanisms and Genes of Cellular Suicide, Science, 267, pp. 1445-1456.

[41] Thompson, C. B. (1995). Apoptosis in the Pathogenesis and Treatment of Disease, Science, 267, pp. 1456-1462.

[42] Zhanggui, W., O'Reilly, M. S., Folkman, J. and Shing, Y. (1997). Suppression of Tumor Growth with Recombinant Murine Angiostatin, Biochemical and Biophysical Research Communications, 236, pp. 651-654. 


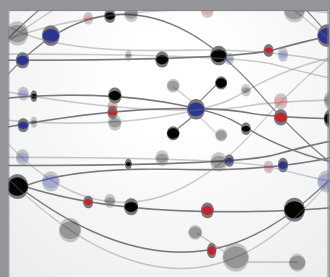

The Scientific World Journal
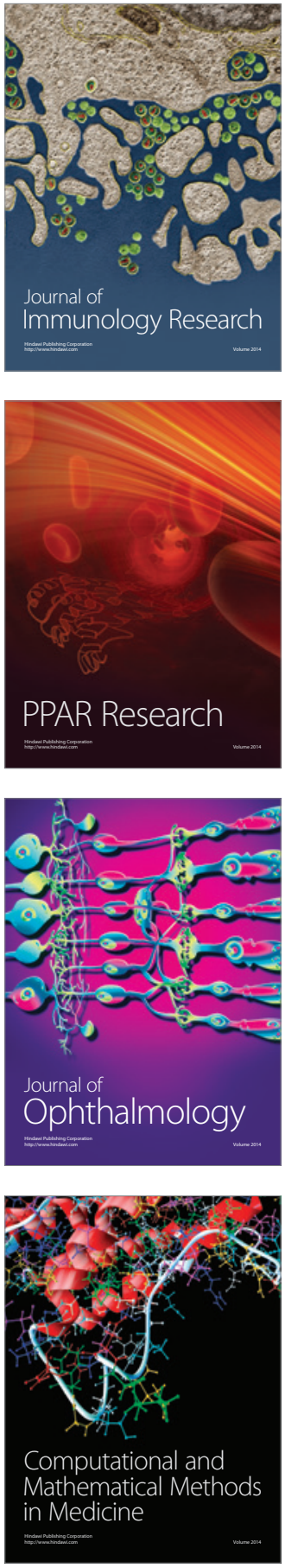

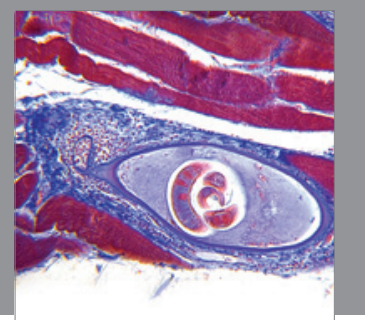

Gastroenterology

Research and Practice
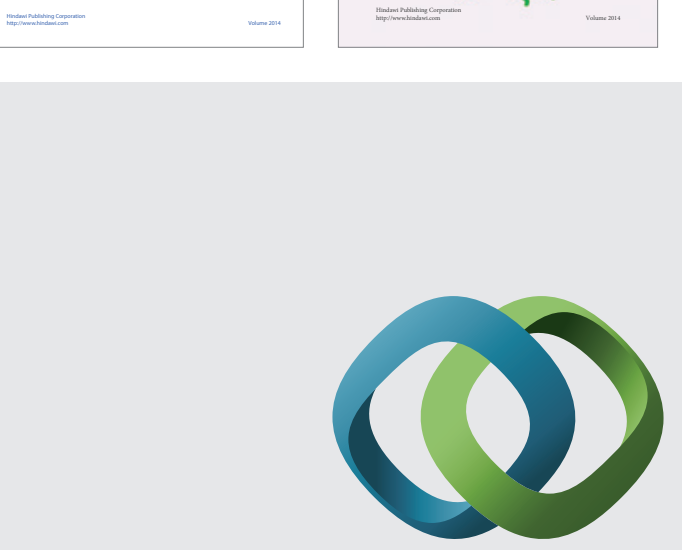

\section{Hindawi}

Submit your manuscripts at

http://www.hindawi.com
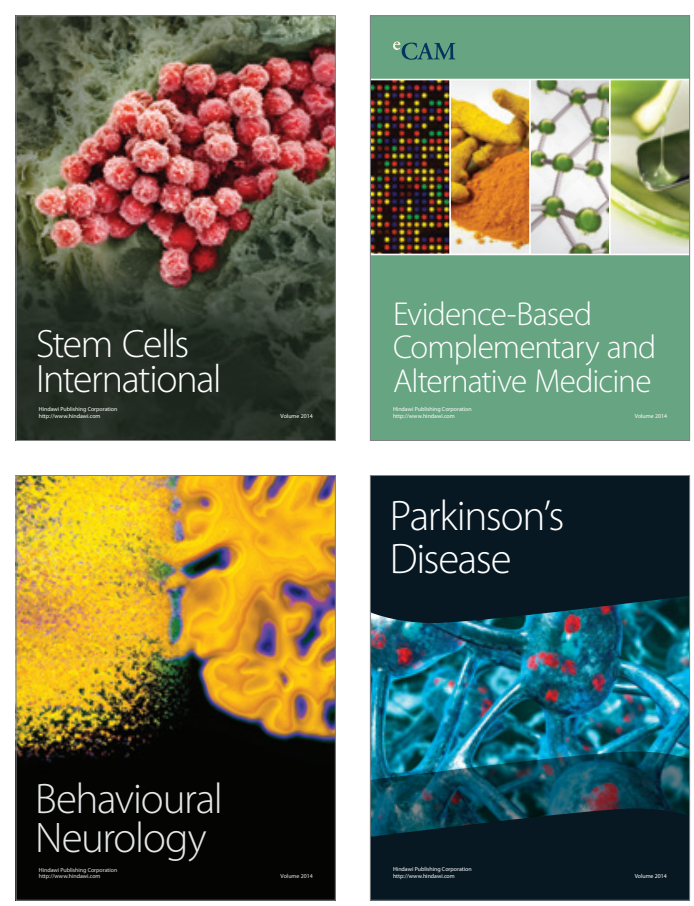

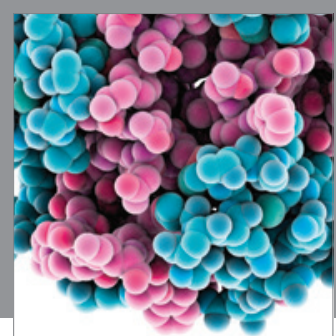

Journal of
Diabetes Research

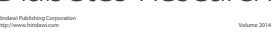

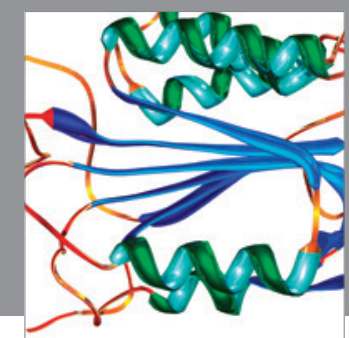

Disease Markers
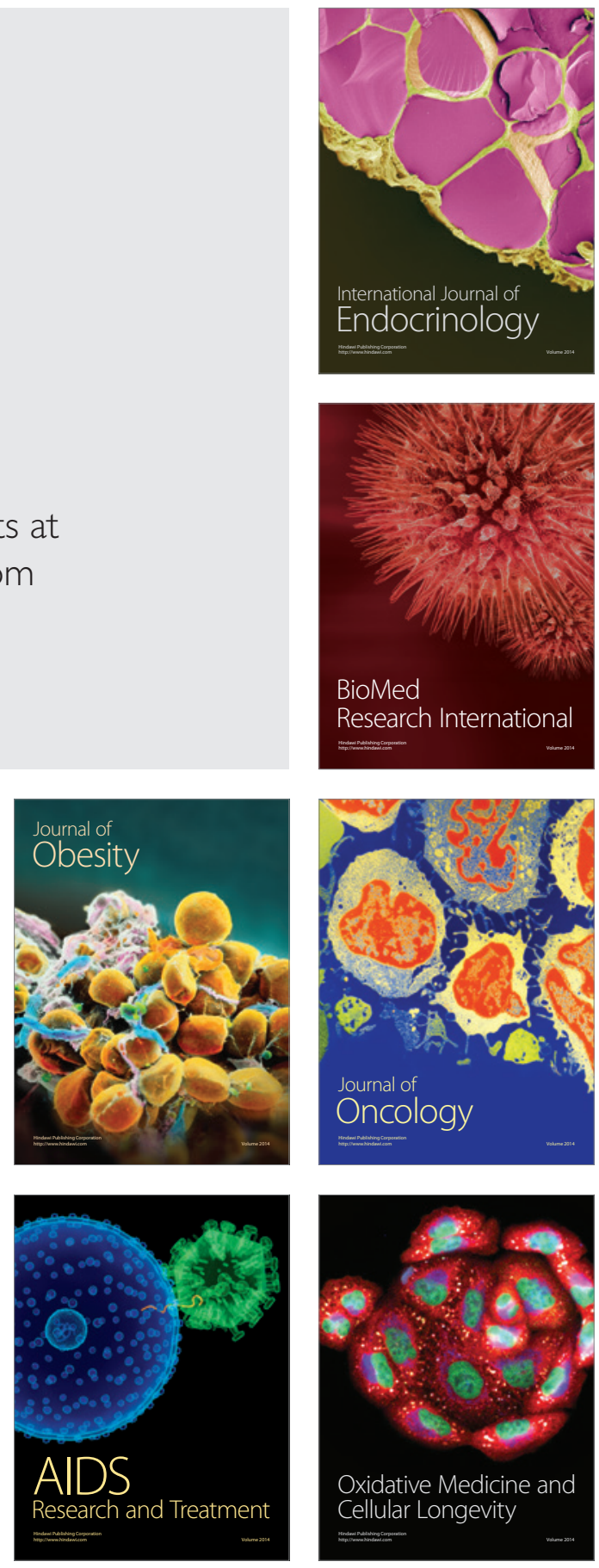